\title{
Gamete adhesion molecules
}

\author{
D. J. Miller and H. R. Burkin \\ Department of Animal Sciences, University of IIlinois at Urbana-Champaign, Urbana, \\ IL. 61801, USA
}

\begin{abstract}
Despite the importance of fertilization for animal production, species preservation and controlling reproduction, the molecular basis underlying fertilization is not well understood. More progress has been made in mice than in other mammals, but targeted deletion of specific genes in the mouse has often yielded unexpected results. The pig is also a useful animal to study, as large numbers of pig gametes can be acquired easily. However, it appears that the pig zona pellucida proteins that bind to spermatozoa may not be homologues of ZP3, the mouse zona pellucida protein that spermatozoa bind to. Therefore, a zona pellucida receptor on spermatozoa that is important for mouse fertilization may be redundant, along with other receptors, in pig fertilization. In this review, the important steps of fertilization in pigs are discussed and the binding of pig gametes is compared with that of mouse gametes. In addition, the molecules that may be important for gamete adhesion are considered. New technical advances and creative ideas offer the opportunity to make important advances in this crucial area.
\end{abstract}

\section{Introduction}

Fertilization is one of the most intriguing biological events. Two haploid cells must attach in a specific manner; this attachment must signal a response from each gamete and the gametes must fuse together. More specifically, the spermatozoon must bind to the extracellular matrix (zona pellucida) of the oocyte, activate the release of a large specialized secretory vesicle (the acrosome) and penetrate this tough extracellular matrix. After penetrating the zona pellucida, the fertilizing spermatozoon must bind to and fuse with the oocyte, resulting in oocyte activation. During activation, the oocyte, previously suspended at metaphase II, completes meiosis and triggers mechanisms to prevent other spermatozoa from penetrating the zona pellucida and causing polyspermy. This complex series of cell interactions allows the formation of a new diploid cell (zygote) that can develop into the wide variety of tissues found in adult animals.

A better understanding of fertilization is vital for improving animal fertility. Once the stages of fertilization are clarified, specific tests to diagnose the causes of reduced fertility and therapies to treat specific causes can be developed. In addition, the fundamental information about fertilization can be used to develop more accurate laboratory tests that allow the identification and removal of subfertile animals from breeding herds. It may also be possible to develop new alternatives to control fertility. Finally, this information can be used to improve the success of in vitro fertilization. 


\section{The zona pellucida}

Oocytes from a variety of vertebrates are surrounded by a glycoprotein coat. In mammals, this relatively thick protective coat is named the zona pellucida. In addition to protecting the developing embryo after fertilization, the zona pellucida binds only spermatozoa from a restricted number of species (Yanagimachi, 1994). Removal of the zona pellucida allows penetration of the oocyte by spermatozoa from a much broader range of species. These observations indicate that specific receptors mediate binding of spermatozoa to the zona pellucida.

Many lines of evidence indicate that the sperm-binding component on the zona pellucida is a carbohydrate. A study by Johnston et al. (1998) showed that the addition of synthetic carbohydrates to spermatozoa blocked the binding of spermatozoa to the zona pellucida. Fixed or denatured zona pellucida can bind spermatozoa, a characteristic not expected if protein is the binding component (Bleil and Wassarman, 1980).

\section{The mouse zona pellucida}

The mouse zona pellucida has been the most extensively studied. The zona pellucida is a product of the oocyte and is composed of three glycoproteins termed ZP1, ZP2 and ZP3, in order of migration on non-reducing SDS polyacrylamide gels. All zona pellucida glycoproteins run as broad bands on gels, due to their extensive and heterogeneous glycosylation. Each protein possesses both asparagine- and serine/threonine-linked oligosaccharides (Wassarman and Litscher, 2001). Related proteins are found in other mammals, and even in birds, amphibians and fish (Rankin and Dean, 2000).

Spermatozoa penetrate the zona pellucida by binding to glycoproteins on the zona pellucida and by releasing the acrosome, an unusual secretory vesicle that is present underneath the plasma membrane and stretched over the anterior region of the sperm nucleus (Yanagimachi, 1994). During the acrosome reaction, the outer acrosomal membrane fuses with the overlying plasma membrane, the contents of the acrosome are released and the inner acrosomal membrane becomes a delimiting sperm membrane. After the acrosome reaction, spermatozoa can penetrate the zona pellucida. Prematurely acrosome-reacted spermatozoa are unable to bind the zona pellucida, possibly because of the loss of the membrane that has the appropriate zona pellucida receptors (Yanagimachi, 1994).

In the fertilization of mice, evidence indicates that one zona pellucida glycoprotein, ZP3, binds acrosome-intact spermatozoa. If the zona pellucida is dissolved and soluble ZP3 is purified, ZP3 binds to acrosome-intact spermatozoa (Wassarman and Litscher, 2001). When spermatozoa are incubated with ZP3 in competitive binding assays, ZP3 blocks binding of spermatozoa to oocytes (Bleil and Wassarman, 1980). However, soluble ZP3 from fertilized eggs does not inhibit binding of spermatozoa to oocytes. The serine/threonine-linked oligosaccharides of ZP3 that bind spermatozoa are apparently modified at fertilization so that they are unable to bind spermatozoa (Florman and Wassarman, 1985).

\section{The pig zona pellucida}

The zona pellucida surrounding pig oocytes has been more difficult to study. The pig zona pellucida is composed of three glycoproteins that have been given various names (Table 1). The pig homologues of ZP1, ZP2 and ZP3 have been most recently termed ssZPB, ssZPA and ssZPC, respectively, in order of the size of the CDNAs encoding the proteins. The molecular weights of ssZPB and ssZPC overlap, a feature that makes their purification by preparative SDS-PAGE impossible (Nakano and Yonezawa, 2001). The only reported procedures for 
Table 1. Nomenclature of vertebrate oocyte coat proteins (adapted from Rankin and Dean, 2000)

\begin{tabular}{lccc}
\hline Species & Egg coat protein I & Egg coat protein II & Egg coat protein III \\
\hline Mouse & ZP1 & ZP2 & ZP3 \\
Pig & ssZPB/ZP3 $\alpha$ & ssZPAZP1 & ssZPC/ZP3 $\beta$ \\
Frog (Xenopus laevis) & & gp69/ZP2/ZPA & gP43/ZP3/ZPC \\
\hline
\end{tabular}

purifying each glycoprotein require partial deglycosylation first. Removal of poly-lactosamine by endo- $\beta$-galactosidase allows separation of ssZPB and ssZPC (Yurewicz et al., 1987; Yonezawa et al., 1995). Although the partially deglycosylated ZPB retains some ability to bind spermatozoa, partial loss of sperm binding activity from ZPB or other zona glycoproteins could go undetected. Therefore, it is difficult to be certain that native ssZPC does not have sperm binding activity. Even ssZPB oligosaccharides that retain some binding activity require molar concentrations two to three orders of magnitude higher than for ssZPB to inhibit sperm-zona binding (Yurewicz et al., 1991).

Considering this caveat, most evidence indicates that the mouse ZP1 homologue, ssZPB (the partially deglycosylated glycoprotein), binds spermatozoa; however, little binding activity has been ascribed to ssZPC, the mouse ZP3 homologue. Later experiments support a cooperative binding model, in which ssZPB requires the presence of some ssZPC to bind spermatozoa (Yurewicz et al., 1998), and this finding is in agreement with the observations that some antibodies to ssZPC block sperm binding (Yurewicz et al., 1998). The sperm binding activity of pig zona pellucida was first ascribed to serine-/threonine-linked oligosaccharides (Yurewicz et al., 1991, 1992), but more recent data are not consistent with that finding. Asparagine-linked oligosaccharides were released from a mixture of ssZPB and ssZPC, and separated into neutral and acidic fractions. Only the neutral asparagine-linked sugars bound spermatozoa (Nakano and Yonezawa, 2001). Unfortunately, the precise oligosaccharide structure that binds spermatozoa was not resolved and there were over 30 different structures in this mixture. Further refinement of analytical approaches shows promise to resolve the oligosaccharide components in zona pellucida glycoproteins (Easton et al., 2000). An advantage of studying the pig zona pellucida is the abundance of material that can be obtained for analysis. The major oligosaccharides in the pig zona pellucida have been described and it is apparent that the carbohydrate chains linked to mouse and pig zona glycoproteins are very different (Easton et al., 2000; Nakano and Yonezawa, 2001).

Most studies attempting to identify the oligosaccharides that bind spermatozoa have used isolated and solubilized whole zonae pellucidae. This approach overlooks the finding that there is a heterogeneous distribution of glycosides within the zona pellucida (Aviles et al., 2000 ). Some monosaccharides (for example, terminal $\alpha$-galactose) cannot be detected by lectin staining in the outer portion of the zona pellucida and are not accessible until a spermatozoon has partially penetrated the zona pellucida (Aviles et al., 2000). Therefore, one must be certain that oligosaccharide candidates proposed as receptors for acrosome-intact spermatozoa are located in the outer portion of the zona pellucida.

\section{Receptors on spermatozoa for the zona pellucida}

In contrast to the zona pellucida, which is a matrix made up of three glycoproteins, the surface of spermatozoa is a much more complex structure. The complexity of the sperm surface is probably one of the reasons why identifying the sperm proteins that bind to the zona pellucida 
has been very challenging. Another complication is that the binding of spermatozoa to the zona pellucida can be divided into two stages. Primary binding refers to the initial step in which acrosome-intact spermatozoa bind to the zona pellucida. After the acrosome reaction, a new membrane domain, the inner acrosomal membrane, is exposed and different receptors may allow spermatozoa to adhere to and penetrate the zona pellucida. Despite these difficulties, a number of candidates for zona pellucida receptors have been proposed, particularly for mouse spermatozoa.

\section{Zona receptors on mouse spermatozoa}

One possible zona pellucida receptor on mouse spermatozoa is sp56, a 56 kDa protein. This protein was first isolated on the basis of its affinity for ZP3 (Cheng et al., 1994). Sequence analysis indicated that sp56 does not have a transmembrane domain (Bookbinder et al., 1995). Recent studies revealed that sp56 is found within the acrosome so that although it cannot be involved in primary binding, it could potentially be exposed during the acrosome reaction and bind as a secondary receptor (Foster et al., 1997). Early studies indicate that acrosome-reacted spermatozoa might bind only ZP2 (Bleil et al., 1988), and this appears to be in conflict with the potential role of sp56 in binding ZP3 after the acrosome reaction. However, it is possible that sp56 binds ZP3 while spermatozoa are completing the acrosome reaction and, after the acrosome reaction is completed, $\mathrm{sp56}$ is lost so that spermatozoa can bind only ZP2. There is evidence that sp56 is exposed gradually and then lost during the relatively slow exocytosis and dispersion of the acrosomal matrix during the acrosome reaction (Kim et al., 2001).

A second possible candidate receptor with a molecular mass of $95 \mathrm{kDa}$ was first identified by its ability to bind ZP3 after SDS-PAGE and transfer to a blot (Leyton and Saling, 1989a). A $95 \mathrm{kDa}$ protein from human testis reported to be a human homologue of the mouse $95 \mathrm{kDa}$ protein is very similar to c-mer (Burks et al., 1995). The human sequence is controversial and the original mouse protein appears to be an unusual hexokinase (Bork, 1996; Kalab et al., 1994; Tsai and Silver, 1996).

The zona receptor whose function has been studied in most detail is $\beta 1,4$ galactosyltransferase (GalTase) (Nixon et al., 2000). This protein was named for the function with which it was first associated, that is, catalysing the addition of galactose to glycoproteins and glycolipids in the Golgi apparatus. More recent studies demonstrated that GalTase is also found on the plasma membrane of some cells, a location enabling it to act as a lectin and bind extracellular glycoconjugates that have $\mathrm{N}$-acetylglucosamine residues presented in the proper context (Nixon et al., 2000). The dual location of this zona pellucida receptor may be explained by the discovery of two forms of GalTase: a full-length form and a short form that results from translation from a downstream start site. The long form has an additional 13 amino acids on its cytoplasmic amino terminus that can apparently override the Golgi retention signal allowing some of the long form to move to the plasma membrane (Youakim et al., 1994).

As a receptor on some cells, GalTase can bind specific extracellular glycoconjugates. GalTase on mouse spermatozoa binds ZP3, but not the other zona pellucida glycoproteins (Miller et al., 1992). Reagents that inhibit GalTase block sperm binding to the zona pellucida (Nixon et al., 2000). The ZP3 oligosaccharides that bind to GalTase, like those that bind to mouse spermatozoa, are linked to ZP3 through serine or threonine residues (Miller et al., 1992). If these GalTase-binding oligosaccharides on ZP3 are removed or blocked, ZP3 does not bind to spermatozoa (Miller et al., 1992).

However, when GalTase is eliminated by homologous recombination, spermatozoa from 
these mice remain fertile, indicating that there may be redundant receptors ( $\mathrm{Lu}$ and Shur, 1997). The ability of spermatozoa from GalTase-null male mice to undergo the acrosome reaction and penetrate the zona pellucida in vitro is severely compromised (Lu and Shur, 1997).

\section{Zona receptors on pig spermatozoa}

Few studies on zona receptors of pig spermatozoa have been reported. Of the receptors described on the surface of mouse spermatozoa, GalTase is the only one that has been examined on pig spermatozoa. GalTase is present on the plasma membrane over the acrosome of pig spermatozoa (Larson and Miller, 1997). Although it binds to pig zona pellucida glycoproteins, GalTase binding is not required for fertilization in pigs. Reagents that inhibit GalTase and block mouse sperm-zona binding do not affect pig sperm-zona binding (Rebeiz and Miller, 1999). As GalTase binds the pig zona pellucida, it may be one of several receptors that function together in gamete binding. However, if other putative receptors are blocked, GalTase alone is not sufficient to allow spermatozoa to adhere to the zona pellucida (Rebeiz and Miller, 1999).

Several other proteins found on pig spermatozoa have been studied. A sperm protein named zonadhesin has affinity for the pig zona pellucida that is species-specific (Hardy and Garbers, 1994). This protein is particularly interesting because it is similar to other proteins that have been implicated in cell-to-cell and cell-matrix interactions (Hardy and Garbers, 1995). If zonadhesin is found within the acrosome, it may function in secondary binding of acrosome-reacted spermatozoa to the zona pellucida. The outcome of studies in which zonadhesin is blocked should be revealing.

There are several peripheral membrane sperm proteins that have been implicated in zona pellucida binding. These proteins may function as adhesive molecules but must couple to other transmembrane proteins to trigger signalling for the acrosome reaction. P47, a protein synthesized by testicular germ cells and the epididymis, has an affinity for the zona pellucida (Ensslin et al., 1998). The consequences of blocking P47 have not been reported. A group of proteins named spermadhesins are produced in the testis, epididymis and accessory sex organs and some of these proteins bind to spermatozoa during epididymal passage or at ejaculation. These proteins have an affinity for a wide variety of ligands, including the zona pellucida (Jansen et al., 2001). As they are peripheral membrane proteins, many are lost during capacitation (Calvete et al., 1997). The necessity of spermadhesins produced by the accessory organs for fertilization is questionable, because removal of these glands does not affect fertility, and spermatozoa removed from the cauda epididymidis are fertile (Davies et al., 1975; Hunter et al., 1976).

One of the difficulties in assessing the function of zona pellucida receptor candidates is that some receptors may function as primary receptors on acrosome-intact spermatozoa, whereas others may act as secondary receptors on acrosome-reacted spermatozoa. The precise location of zona pellucida receptors on spermatozoa was addressed by labelling zona pellucida proteins directly with a bright fluorochrome and allowing labelled zona pellucida proteins to bind pig spermatozoa (Burkin and Miller, 2000). Dead spermatozoa were labelled with propidium iodide. Motile spermatozoa that excluded propidium iodide showed zona pellucida protein staining at the apical edge of spermatozoa (Fig. 1). No staining was observed when control proteins were labelled and incubated with spermatozoa. The staining with pig zona pellucida proteins was specific and saturable. The addition of excess unlabelled zona pellucida proteins displaced binding of labelled zona pellucida proteins.

No difference was observed in the labeling of capacitated compared with washed uncapacitated spermatozoa (Burkin and Miller, 2000). In contrast, when spermatozoa were 

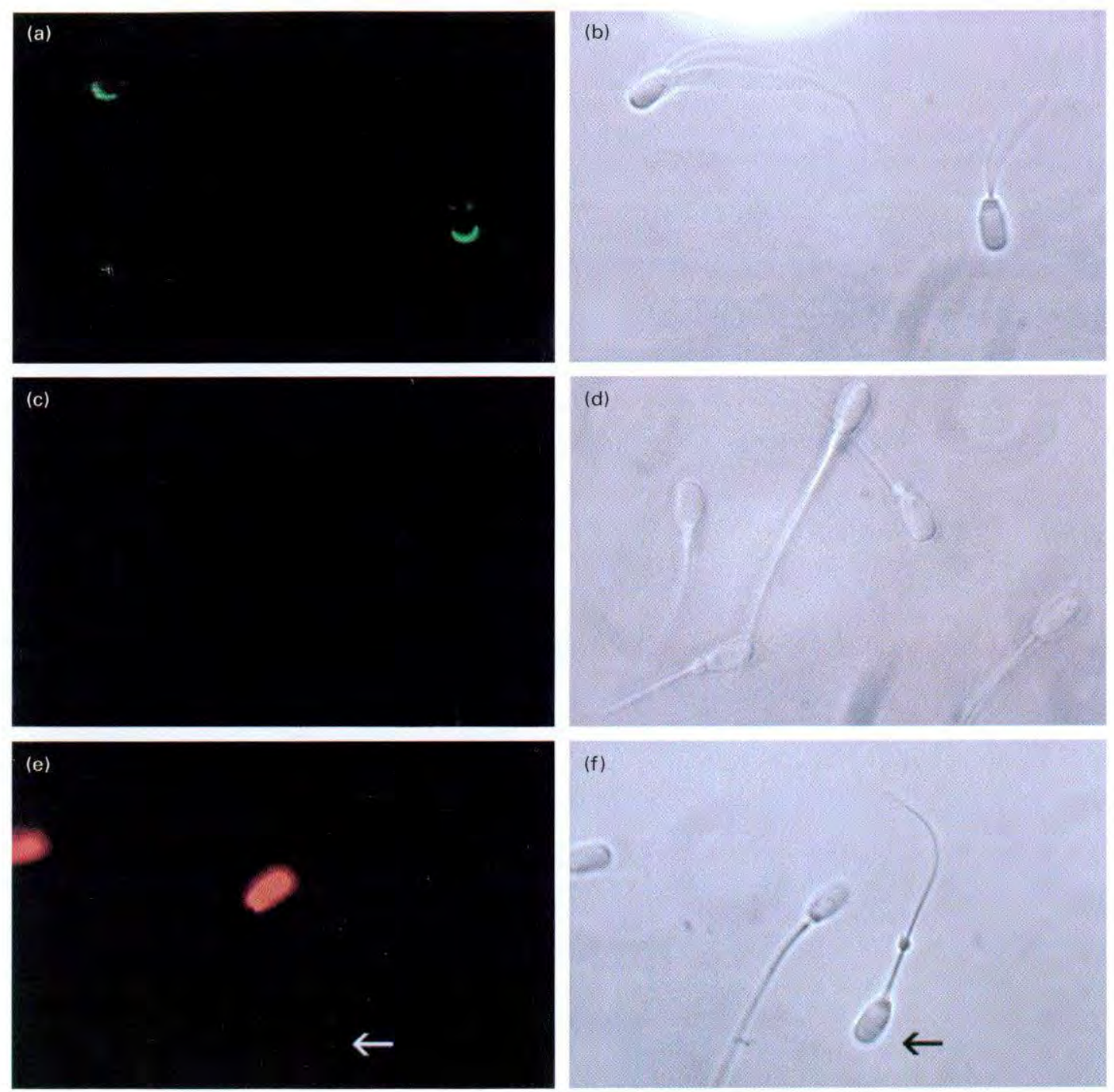

Fig. 1. Localization of solubilized zona pellucida proteins on live spermatozoa. Zona pellucida proteins were labelled with Alexa, a green fluorochrome. Labelled proteins and propidium iodide were added to live pig spermatozoa. Alexa-labelled zona pellucida proteins bound live, acrosome-intact spermatozoa over the anterior head region concentrated over the acrosomal ridge (a). The addition of a 100 -fold excess of unlabelled zona pellucida proteins displaced the signal (c). The Alexa-labelled control glycoprotein, transferrin, did not bind to live spermatozoa indicated by the arrow $(e, f)$. Dead spermatozoa stained with propidium iodide are visible because fluorescence images were captured using a filter set that allowed detection of both red and green fluorochromes simultaneously $(\mathrm{a}, \mathrm{c}, \mathrm{e})$. Corresponding phase-contrast images are shown $(\mathrm{b}, \mathrm{d}, \mathrm{f})$.

treated with the calcium ionophore A23187 to induce the acrosome reaction, zona pellucida protein binding was altered markedly. The entire apical half of the sperm head, including the region from the tip of the acrosome to the equatorial region, bound zona pellucida proteins (Fig. 2). This matched the region that bound zona pellucida proteins on fixed spermatozoa, probably because acrosomal zona pellucida binding proteins were exposed by fixation.

On the basis of these results, we predict that proteins on acrosome-intact spermatozoa 
involved in primary binding are located mainly on the plasma membrane overlying the apical ridge of the acrosome. The receptors on acrosome-reacted spermatozoa that are important for secondary binding are expected to be located over the entire acrosomal area of acrosomereacted or fixed spermatozoa. Several of the receptor candidates studied appear to be located over the entire acrosomal area and, on the basis of zona protein binding, would be stronger candidates for secondary receptors than for primary receptors (Mori et al., 1995; Ensslin et al., 1998).

There are several candidates that may act as secondary receptors for acrosome-reacted spermatozoa, but there are fewer compelling candidates for zona pellucida receptors on acrosome intact spermatozoa. There are now more sophisticated biological tests of the function of putative zona pellucida receptors, such as gene knockouts in mice, a technique that will eventually be more common in other mammals. New approaches or a re-evaluation of the current approaches should enable additional candidates for primary zona receptors to be isolated.

\section{The acrosome reaction and penetration of zona pellucida}

Spermatozoa must undergo the exocytotic process, known as the acrosome reaction, to penetrate the zona pellucida. In mouse spermatozoa, acrosomal exocytosis is activated by ZP3 binding (Wassarman and Litscher, 2001). Although the oligosaccharides bind spermatozoa, the intact ZP3 glycoprotein is required to induce the acrosome reaction (Florman et al., 1984). Interestingly, glycopeptides derived from pronase treatment of ZP3 can induce the acrosome reaction if a 'backbone' is provided by an antibody (Leyton and Saling, $1989 \mathrm{~b})$. This result led to the hypothesis that the function of the ZP3 protein backbone is to provide a scaffold for the sperm-binding oligosaccharides. Alternatively, the protein backbone could interact with a unique receptor that triggers the acrosome reaction.

ZP3 may trigger the acrosome reaction by activating the receptor that is important in initial binding. Alternatively, ZP3 may trigger the acrosome reaction by interacting with a second lower affinity receptor that is more important for signal transduction than initial binding. The only ZP3 receptor demonstrated to have a role in signal transduction is GalTase. Some GalTase antibodies are capable of clustering GalTase in a manner that appears to mimic ZP3 and trigger the acrosome reaction (Macek et al., 1991). GalTase can bind two to three ZP3 molecules and some GalTase antibodies also appear to bind GalTase multivalently (Miller et al., 1992). Monovalent Fab fragments do not induce the acrosome reaction but if the Fab fragments are made multivalent by addition of a second antibody, this complex induces the acrosome reaction. Aggregation of GalTase with antibodies activates heterotrimeric $G$ proteins and triggers the acrosome reaction in a manner requiring pertussis toxin-sensitive G proteins (Gong et al., 1995). This result is surprising as most $G$ protein-coupled receptors have seven transmembrane domains and GalTase has only one. However, GalTase has clusters of basic amino acid residues that are necessary for $\mathrm{G}$ protein activation by traditional and non-traditional $G$ protein-coupled receptors. At least one of these clusters of basic amino acid residues is required for $G$ protein activation and GalTase signalling (Shi et al., 2001).

An important role for GalTase in the acrosome reaction was confirmed by deleting GalTase by homologous recombination. Although spermatozoa from GalTase null mice bind to the zona pellucida, they acrosome react and penetrate the zona pellucida with only $7 \%$ of the frequency of control spermatozoa (Lu and Shur, 1997).

After the acrosome reaction, spermatozoa must remain stable on the zona pellucida in the secondary binding step. Some of the candidate receptors described above that have zona affinity and are found within the acrosome are good candidates for secondary receptors. 
(a)
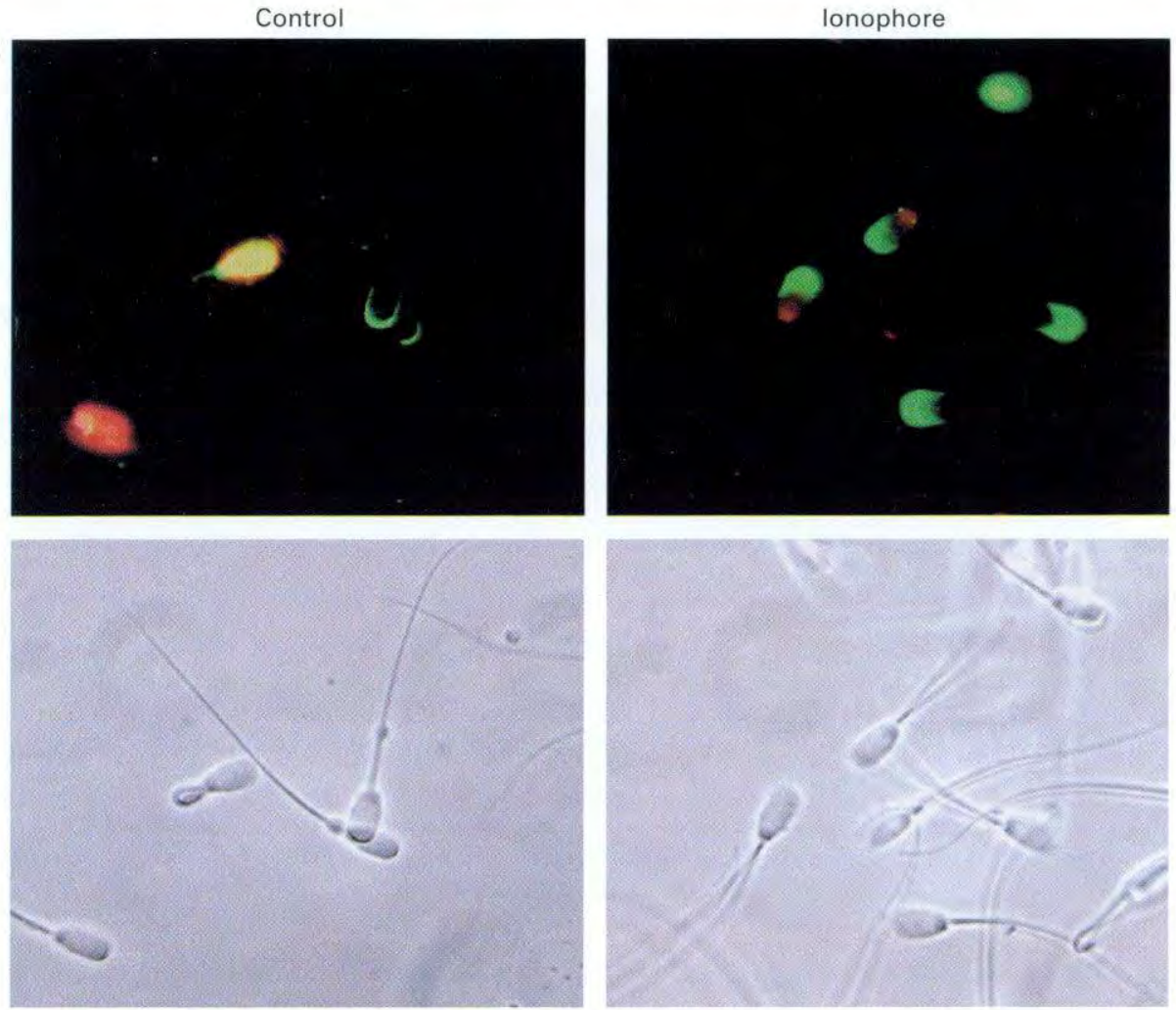

(b)

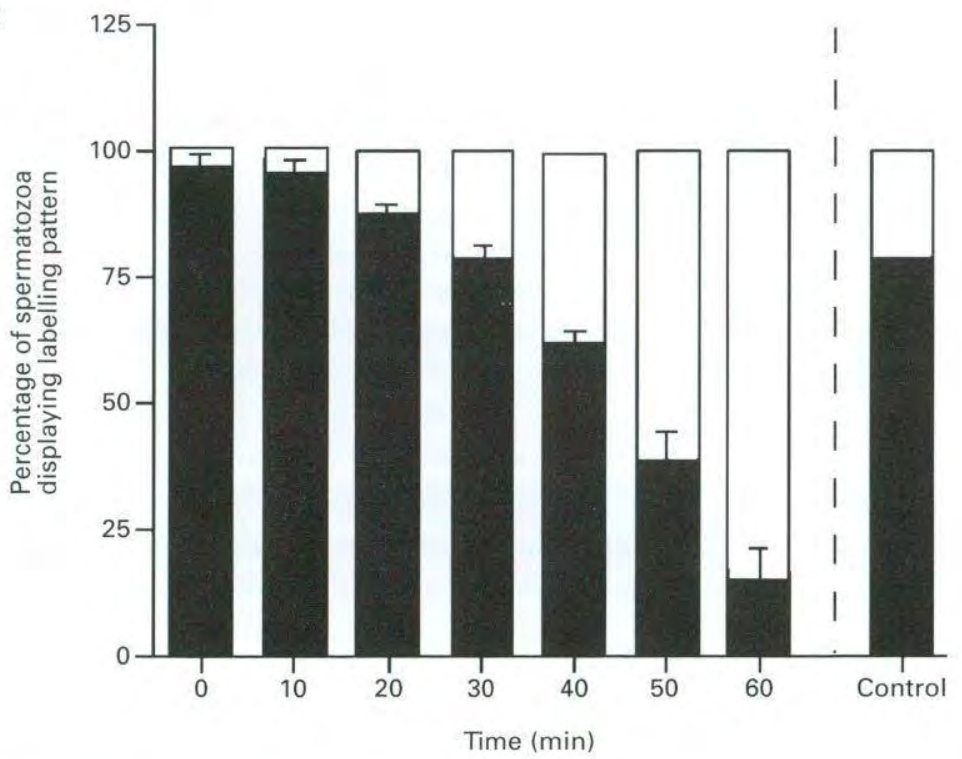

Fig. 2. For legend see opposite. 
These candidates may function sequentially as the acrosome is exocytosed and the matrix is slowly dispersed. After the acrosome reaction, spermatozoa begin to penetrate the zona pellucida. This process requires a specialized hyperactivated motility to generate force to move through the zona pellucida (Yanagimachi, 1994). It also requires a localized dissolution of the zona pellucida, creating a penetration slit. There is considerable debate about whether zona penetration requires some hydrolysis of zona pellucida proteins or, apparently like penetration of the sea abalone egg coat (Kresge et al., 2001), penetration does not require hydrolysis (Bedford, 1998).

In mice, there is evidence that ZP2 binds to acrosome-reacted spermatozoa to maintain spermatozoa on the zona pellucida (Bleil et al., 1988). It is possible that ZP2 binds to components of the sperm acrosome, such as proacrosin and other proteases, to mediate secondary binding. Proacrosin is the zymogen form of acrosin, a serine protease that is abundant in the sperm acrosome (Jansen et al., 2001). Proacrosin has affinity for sulphated fucose and sulphated zona pellucida proteins. The zona pellucida binding activity and enzyme activity of proacrosin are located in different regions of the molecule, and it is possible that both regions work in concert in adhesion and lysis of the zona pellucida during zona pellucida penetration. Gene knockout studies demonstrated that spermatozoa lacking proacrosin, although still fertile, are at a disadvantage compared with spermatozoa with normal amounts of proacrosin (Adham et al., 1997). Another protein involved in secondary binding is $\mathrm{PH}-20$, a sperm hyaluronidase that appears to have two functions. $\mathrm{PH}-20$, present on the posterior head membrane of spermatozoa, can cleave hyaluronic acid in the matrix surrounding oocytes and, after the acrosome reaction, appears to be important for binding the zona pellucida (Myles and Primakoff, 1997). Blocking $\mathrm{PH}-20$ prevents zona pellucida penetration (Myles and Primakoff, 1997; Yudin et al., 1999).

\section{Fusion of spermatozoa with the oocyte plasma membrane}

Once inside the zona pellucida, spermatozoa bind to, and fuse with, the oocyte plasma membrane. A monoclonal antibody that inhibited sperm fusion to the plasma membrane of the oocyte provided the first step in identifying these adhesion molecules. This antibody reacted with a dimeric protein termed fertilin (Blobel et al., 1992). Fertilin is a member of a growing family of molecules called ADAMs for their disintegrin and metalloprotease domains (adhesion, disintegrin and metalloprotease). The disintegrin domain is believed to interact with integrins on the oocyte membrane, specifically $\alpha 6 \beta 1$ (Almeida et al., 1995). Studies in

Fig. 2. Region of spermatozoa that binds zona pellucida proteins is increased in acrosome-reacting spermatozoa. Live, capacitated boar spermatozoa were incubated with the calcium ionophore A23187 to induce the acrosome reaction, followed by the addition of Alexa-labelled zona pellucida proteins and propidium iodide. The upper panels in (a) show fluorescence images of Alexa-labelled zona pellucida and propidium iodide, and the lower panels show corresponding phase-contrast images. Acrosome-reacted spermatozoa showed an increased area of zona pellucida protein binding, extending from the acrosomal ridge to the equatorial region of live spermatozoa, whereas controls without ionophore did not. For time course experiments (b), samples were removed at the specified times after the addition of ionophore. The percentage of live spermatozoa displaying zona pellucida binding in a thin band over the acrosomal ridge decreased with time (black bars), while the percentage displaying strong acrosomal fluorescence increased (white bars). Controls to which ionophore was not added were counted after $60 \mathrm{~min}$. The graph shows the mean percentage of spermatozoa \pm standard error. Regression analysis indicated a significant increase in the percentage of spermatozoa displaying strong fluorescence over the entire acrosomal region that was correlated with induction of the acrosome reaction. 
mice with deletions in these genes produced by homologous recombination have yielded unexpected results. Spermatozoa from mice lacking fertilin- $\beta$ have defects in transport through the oviduct and zona binding, but some spermatozoa can still fuse and activate oocytes (Cho et al., 1998). Spermatozoa from knockouts of more recently described members of the ADAMs family were also defective in zona binding, but were not defective in oocyte membrane binding (Shamsadin et al., 1999). As the ligand for these ADAMs family members was believed to be the integrin dimer $\alpha 6 \beta 1$, oocytes from animals lacking $\alpha 6$ were examined. These oocytes had no apparent defects in fertilization (Miller et al., 2000). There may be redundant receptors that bind spermatozoa to the oocyte plasma membrane.

There are few studies of pig sperm binding to the oocyte plasma membrane. Pig oocytes express $\alpha v$ and $\beta 1$ integrins on the surface membrane (Linfor and Berger, 2000). Several candidate ligands, described by their affinity for the oocyte membrane and their migration on SDS-PAGE, have been reported (Linfor and Berger, 2000; Sartini and Berger, 2000). Further studies to determine the biological importance of these adhesion molecules are necessary.

\section{Egg activation and the block to polyspermy}

After the spermatozoon binds and fuses with the oocyte plasma membrane, there is a release of calcium, a hallmark of fertilization (Yanagimachi, 1994). In mammals, there is a series of calcium oscillations that may be triggered by a sperm component that is released in the oocyte (Carroll, 2001). The cortical granules are released, the oocyte resumes and completes meiosis, and the sperm nucleus decondenses. As part of the block to polyspermy, the contents of the cortical granules act on the overlying zona pellucida. An enzyme that hydrolyses mouse ZP2 into two components is released, although it is not known how this proteolysis affects the function of ZP2 (Moller and Wassarman, 1989). A second enzyme, hexosaminidase B, is released from cortical granules during oocyte activation and acts on ZP3 (Miller et al., 1993). This enzyme removes the terminal monosaccharide, $\mathrm{N}$-acetylglucosamine, that binds GalTase. If hexosaminidase B is inhibited, polyspermic binding occurs (Miller et al., 1993). There are few molecular studies of the block to polyspermic binding in pig oocytes, despite the high rate of polyspermy with in vitro fertilization in pigs.

\section{Conclusion}

Despite the importance of fertilization, a complete understanding of the receptors involved in gamete binding and fusion at the molecular level remains to be elucidated. The difficulty in obtaining sufficient material has made it problematic to determine the oligosaccharides from the zona pellucida that bind spermatozoa. Although it is apparent that soluble ZP3 is the only protein that binds acrosome-intact mouse spermatozoa, the zona pellucida protein that binds pig spermatozoa may not be the ZP3 homologue, but instead may be the homologue of mouse ZP1, known as ZPB. The complementary receptors for zona pellucida proteins on spermatozoa must be clarified. Studies of the identified receptor candidates must be completed and further studies are required to investigate new candidates. It is not clear how zona pellucida proteins are inactivated when the oocyte is fertilized and this causes difficulties for in vitro fertilization in pigs because of the high rates of polyspermy. New analytical techniques and novel approaches hold great promise for identifying molecules important for fertilization. The ability to modify animal genomes should enable the functions of old and new fertilization proteins to be tested and should help to resolve this enigma. 
The authors apologize to those whose work was not cited due to space limitations. Work in the authors' laboratory was supported by the USDA National Research Initiative Competitive Grants Program, the National Science Foundation, and the National Institutes of Health.

\section{References}

Adham IM, Nayernia $K$ and Engel W (1997) Spermatozoa lacking acrosin protein show delayed fertilization Molecular Reproduction and Development 46 370-376

Almeida EAC, Huovila A-PI, Sutherland AE et al. (1995) Mouse egg integrin $\alpha 6 \beta 1$ functions as a sperm receptor Cell 81 1095-1104

Aviles M, Okinaga T, Shur BD and Ballesta J (2000) Differential expression of glycoside residues in the mammalian zona pellucida Molecular Reproduction and Development 57 296-308

Bedford JM (1998) Mammalian f́ertilization misread? Sperm penetration of the eutherian zona pellucida is unlikely to be a lytic event Biology of Reproduction 59 1275-1287

Bleil JD and Wassarman PM (1980) Mammalian sperm-egg interaction: identification of a glycoprotein in mouse egg zonae pellucidae possessing receptor activity for sperm Cell 20 873-882

Bleil JD, Greve JM and Wassarman PM (1988) Identification of a secondary sperm receptor in the mouse egg zona pellucida: role in maintenance of binding of acrosomereacted sperm to eggs Developmental Biology 128 376-385

Blobel CP, Wolfsberg TC, Turck CW, Myles DG, Primakoff $P$ and White JM (1992) A potential fusion peptide and an integrin ligand domain in a protein active in sperm-egg fusion Nature 356 248-252

Bookbinder LH, Cheng A and Bleil JD (1995) Tissue- and species-specific expression of $\mathrm{sp56}$, a mouse sperm fertilization protein Science 269 86-89

Bork P (1996) Sperm-egg binding protein or protooncogene? Science 271 1431-1432

Burkin HR and Miller DJ (2000) Zona pellucida protein binding ability of pig sperm during epididymal maturation and the acrosome reaction Developmental Biology 222 99-109

Burks DJ, Carballada R, Moore HDM and Saling PM (1995) Interaction of a tyrosine kinase from human sperm with the zona pellucida at fertilization Science $26983-86$

Calvete II, Ensslin M, Mburu I et al. (1997) Monoclonal antibodies against boar sperm zona pellucida-binding protein AWN-1. Characterization of a continuous antigenic determinant and immunolocalization of AWN epitopes in inscminated sows Biology of Reproduction 57 735-742

Carroll I (2001) The initiation and regulation of $\mathrm{Ca}^{2+}$ signalling at fertilization in mammals Seminars in Cell and Developmental Biology $1237-43$

Cheng A, Le T, Palacios M, Bookbinder LH, Wassarman PM, Suzuki $F$ and Bleil JD (1994) Sperm-egg recognition in the mouse: characterization of sp56, a sperm protein having specific affinity for ZP3 lournal of Cell Biology $125867-878$

Cho C, Bunch DO, Faure JE, Goulding EH, Eddy EM, Primakoff P and Myles DC (1998) Fertilization defects in sperm from mice lacking fertilin $\beta$ Science 281 1857-1859

Davies DC, Hall G, Hibbitt G and Moore HD (1975) The removal of the seminal vesicles from the boar and the effects on the semen characteristics Journal of Reproduction and Fertility 43 305-312

Easton RL, Patankar MS, Lattanzio FA, Leaven TH, Morris HR, Clark GF and Dell A (2000) Structural analysis of murine zona pellucida glycans. Evidence for the expression of core 2-type O-glycans and the $\mathrm{Sd}^{3}$ antigen journal of Biological Chemistry 275 7731-7742

Ensslin M, Vogel T, Calvete IJ, Thole $\mathbf{H H}$, Schmidtke J, Matsuda $T$ and Topfer-Petersen E (1998) Molecular cloning and characterization of $\mathrm{P} 47$, a novel boar spermassociated zona pellucida-binding protein homologous to a family of mammalian secretory proteins Biology of Reproduction 58 1057-1064

florman HM and Wassarman PM (1985) O-linked oligosaccharides of mouse egg ZP3 account for its sperm receptor activity Cell 41 313-324

Florman HM, Bechtol KB and Wassarman PM (1984) Enzymatic dissection of the functions of the mouse egg's receptor function for sperm Developmental Biology 106 $243-255$

Foster J, Friday B, Maulit M, Blobel C, Winfrey V, Olson C, Kim $K$ and Gerton G (1997) AM67, a secretory component of the guinea pig sperm acrosomal matrix, is related to mouse sperm protein $s p 56$ and the complement component 4 -binding proteins fournal of Biological Chemistry 27212 714-12 722

Gong XH, Dubois DH, Miller D) and Shur BD (1995) Activation of a $G$ protein complex by aggregation of $\beta-1,4$ galactosyltransferase on the surface of sperm Science 269 1718-1721

Hardy DM and Garbers DL (1994) Species-specific binding of sperm proteins to the extracellular matrix (zona pellucida) of the cgg Journal of Biological Chemistry 269 19000-19004

Hardy DM and Garbers DL (1995) A sperm membrane protein that binds in a species-specific manner to the egg extracellular matrix is homologous to von Willebrand factor Journal of Biological Chemistry 270 26 025-26028

Hunter RH, Holtz W and Henfrey PJ (1976) Epididymal function in the boar in relation to the fertilizing ability of spermatozoa Journal of Reproduction and Fertility 46 $463-466$

Jansen S, Ekhlasi-Hundrieser $M$ and Topfer-Petersen $E$ (2001) Sperm adhesion molecules: structure and function Cells, Tissues, Organs 168 82-92

Johnston DS, Wright WW, Shaper JH, Hokke CH, Van den Eijnden DH and Joziasse DH (1998) Murine sperm-zona binding, a fucosyl residue is required for a high affinily sperm-binding ligand Journal of Biological Chemistry 273 1888-1895 
Kalab P, Visconti P, Leclerc P and Kopf GS (1994) p95, the major phosphotyrosine-containing protein in mouse spermatozoa, is a hexokinase with unique properties Journal of Biological Chemistry $2693810-3817$

Kim KS, Foster JA and Gerton GL (2001) Differential release of guinea pig sperm acrosomal components during exocytosis Biology of Reproduction 64 148-156

Kresge N, Vacquier VD and Stout CD (2001) Abalone lysin: the dissolving and evolving sperm protein Bioessays $\mathbf{2 3}$ 95-103

Larson JL and Miller DJ (1997) Sperm from a variety of mammalian species express $\boldsymbol{\beta} 1,4$-galactosyltransferase on their surface Biology of Reproduction 57 442-453

Leyton L and Saling P (1989a) $95 \mathrm{kd}$ sperm proteins bind ZP3 and serve as tyrosine kinase substrates in response to zona binding Cel/57 1123-1130

Leyton L and Saling P (1989b) Evidence that aggregation of mouse sperm receptors by $Z P 3$ triggers the acrosome reaction Journal of Cell Biology 108 2163-2168

Linfor J and Berger T (2000) Potential role of $\alpha v$ and $\beta 1$ integrins as oocyte adhesion molecules during fertilization in pigs fournal of Reproduction and Fertility 120 65-72

Lu $Q$ and Shur BD (1997) Sperm from B1,4. galactosyltransíerase-null mice are refractory to $\mathrm{ZP} 3$ induced acrosome reaclion and penetrate the zona pellucida poorly Development 124 4121-4131

Macek MB, Lopez LC and Shur BD (1991) Aggregation of $\beta$ 1,4-galactosyltransferase on mouse sperm induces the acrosome reaction Developmental Biology $147440-444$

Miller BJ, Georges-Labouesse E, Primakoff $P$ and Myles DG (2000) Normal fertilization occurs with eggs lacking the integrin $\alpha 6 \beta 1$ and is CD9-dependent fournal of Cell Biology 149 1289-1296

Miller DJ, Gong XH, Decker G and Shur BD (1993) Egg cortical granule $\mathrm{N}$-acetylglucosaminidase is required for the mouse zona block to polyspermy journal of $\mathrm{Cell}$ Biology 123 1431-1440

Miller DJ, Macek MB and Shur BD (1992) Complementarity between sperm surface $\boldsymbol{\beta}$-1,4-galactosyltransferase and egg-coat ZP3 mediates sperm-egg binding Nature 357 589-593

Moller CC and Wassarman PM (1989) Characterization of a proteinase that cleaves zona pellucida glycoprotein ZP2 following activation of mouse eggs Developmental Biology 132 103-112

Mori E, Kashiwabara S, Baba T, Inagaki $Y$ and Mori $T$ (1995) Amino acid sequences of pig Sp38 and proacrosin required for binding to the zona pellucida Developmental Biology $168575-583$

Myles DG and Primakoff P (1997) Why did the sperm cross the cumulus? To get to the oocyte. Functions of the sperm surface proteins $\mathrm{PH}-20$ and fertilin in arriving at, and fusing with, the egg Biology of Reproduction 56 320-327

Nakano $M$ and Yonezawa $N$ (2001) Localization of sperm ligand carbohydrate chains in pig zona pellucida glycoproteins Cells, Tissues, Organs 168 65-75

Nixon B, Lu Q, Wassler M, Foote C, Ensslin M and Shur BD (2000) Galactosyltransferase function during mammalian fertilization Cells, Tissues, Organs 168 46-57
Rankin T and Dean J (2000) The zona pellucida: using molecular genelics to study the mammalian egg coat Reviews of Reproduction 5 114-121

Rebeiz $M$ and Miller DJ (1999) Pig sperm surface $\beta 1,4$ galactosyltransferase binds to the zona pellucida but is not necessary or sufficient to mediate spermzona pellucida binding Molecular Reproduction and Development 54 379-387

Sartini BL and Berger T (2000) Identification of homologous binding proteins in pig and bovine gametes Molecular Reproduction and Development 55 446-451

Shamsadin R, Adham IM, Nayernia K, Heinlein UA, Obenwinkler $H$ and Engel $W$ (1999) Male mice deficient for germ-cell cyritestin are infertile Biology of Reproduction 61 1445-1451

Shi X, Amindari S, Paruchuru K, Skalla D, Burkin H, Shur $B D$ and Miller DJ (2001) Cell surface $\beta-1,4$ galactosyltransferase-I activates $G$ protein-dependent exocytotic signaling Development 128 645-654

Tsai J-Y and Silver L (1996) Sperm-egg binding protein or proto-oncogene Science 271 1431-1432

Wassarman PM and Litscher ES (2001) Towards the molecular basis of sperm and egg interaction during mammalian fertilization Cells, Tissues, Organs 168 $36-45$

Yanagimachi $R$ (1994) Mammalian fertilization. In Physiology of Reproduction, 2nd Edn A1 89-A317 Eds E Knobil and JD Neill. Raven Press, New York

Yonezawa N, Aoki H, Hatanaka $Y$ and Nakano $M$ (1995) Involvement of $\mathrm{N}$-linked carbohydrate chains of pig zona pellucida in sperm-egg binding European Journal of Biochemistry 233 35-41

Youakim A, Dubois D and Shur B (1994) Localization of the long form of $\beta$-1,4-galactosyltransierase to the plasma membrane and Golgi complex of 3T3 and F9 cells by immunofluorescence coníocal microscopy Proceedings National Academy of Sciences USA 91 10913-10917

Yudin Al, Vandevoorl CA, Li MW and Overstreet IW (1999) $\mathrm{PH}-20$ but not acrosin is involved in sperm penetration of the macaque zona pellucida Molecular Reproduction and Development 53 350-362

Yurewicz EC, Sacco AG and Subramanian MG (1987) Structural characterization of the $M_{r}=55,000$ antigen (ZP3) of pig oocyte zona pellucida Journal of Biological Chemistry 262 564-571

Yurewicz EC, Pack BA and Sacco AG (1991) Isolation, composition and biological activity of sugar chains of pig oocyte zona pellucida $55 \mathrm{~K}$ glycoproteins Molecular Reproduction and Development 30 126-134

Yurewicz EC, Pack BA and Sacco AG (1992) Pig oocyte zona pellucida $\mathrm{Mr} \mathbf{5 5 , 0 0 0}$ glycoproteins: identification of O-glycosylated domains Molecular Reproduction and Development 33 182-188

Yurewicz EC, Sacco AG, Gupta SK, Xu N and Gage DA (1998) Hetero-oligomerization-dependent binding of pig oocyte zona pellucida glycoproteins ZPB and ZPC to boar sperm membrane vesicles Journal of Biological Chemistry 273 7488-7494 